\title{
PRODUCCIÓN Y USO DE LA CERÁMICA VALDIVIA FASE VIII (COMPLEJO PIQUIGUA), DEL SITIO SAN ISIDRO NORTE DE MANABÍ, ECUADOR
}

\author{
MANUFACTURE AND USE OF THE VALDIVIA PHASE VIII CERAMIC \\ (PIQUIGUA COMPLEX), IN SAN ISIDRO NORTH OF MANABÍ, ECUADOR
}

\begin{abstract}
RESUMEN
En este artículo, se revisa estadísticamente la dependencia entre la producción y el uso de la cerámica Valdivia Terminal. La información que se utiliza pertenece al Complejo Piquigua, Fase VIII de la Cultura Valdivia del sitio San Isidro, Norte de Manabí (Jadán 1986). Se estudia las formas de las vasijas en relación con dos variables. Por una parte, el tamaño del desgrasante y por otra los acabados o tratamientos realizados en los recipientes. El análisis se ejecuta con microscopio binocular en una muestra de 368 fragmentos entre, bordes, bases y cuerpos de vasijas. Mediante el examen de diez muestras de láminas delgadas de la cerámica, se ratifica la información obtenida en el microscopio binocular. Se observa las huellas dejadas en la superficie de los tiestos, lo que facilita el conocimiento de las técnicas, artificios e instrumentos utilizados en esta etapa de elaboración de las vasijas.
\end{abstract}

Palabras clave: acabado de superficie; forma; función; producción; tamaño del desgrasante.

\section{ABSTRACT}

In this article, Valdivia Terminal is statistically revised, its dependence between the manufacture and the use of ceramics. The collected information belongs to the Piquigua complex, phase VIII of the Valdivia culture of San Isidro, North of Manabi (Jadán 1986) site. The vessels shapes are analyzed in relation with two variables. On one hand, the size of degreasing and by the other the treatments and processing carried out in vessels. The analysis runs with binocular microscope in a sample of 368 segments among, bases, sides and bodies of pottery fragments. By examining ten samples of thin sheets of ceramic, is confirmed the gotten information from the binocular microscope. Is observed the footprints left on the surface of the pots, which facilitates the knowledge of the techniques, devices and instruments used in this stage of development of the vessels.

Keywords: function; manufacture; processing; shape; size of degreasing.

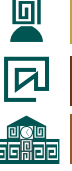




\section{INTRODUCCIÓN}

Valdivia es una de las culturas del Formativo Ecuatoriano que mayor atención ha recibido por parte de los estudiosos. A partir de su descubrimiento por Emilio Estrada (1956), se han ejecutado numerosas investigaciones entre las que mencionaremos los estudios realizados por Meggers, Evans y Estrada (1965); Zevallos y Holm (1960); Zevallos (1971); Norton (1982); Lathrap, Marcos y Zeidler (1977); Zeidler (1994); entre otros. Meggers et al. (1965), plantearon que los Valdivia eran pueblos pescadores y recolectores de moluscos y que su cerámica fue introducida por pescadores del Neolítico Japonés en un desembarco accidental en la costa ecuatoriana. Posteriores estudios realizados por Zevallos y Holm (1960) en el sitio San Pablo, así como el hallazgo de un grano de maíz parcialmente carbonizado en un tiesto perteneciente a Valdivia Medio (Zevallos 1971), sugirieron que los Valdivia fueron sociedades agrícolas.

Las investigaciones realizadas por Norton (1982), en el sitio San Pablo, sitio orientado en los valles costeños y alejado del mar, apoyó una base agrícola, pero además evidenció a la sociedad Valdivia, con una de las cerámicas más antiguas de América. En 1974 - 1975, la Universidad de Illinois realizó una investigación en el sitio Real Alto, se obtuvieron resultados muy fructíferos sobre economía, organización social y ritualismo (Lathrap et al. 1977).

En los años 1981 a 1983 se ejecutó el Proyecto «Sociedad Prehistórica e Intercambio Regional en el Sitio San Isidro, Manabí»». Fue realizado por la Escuela Superior Politécnica del litoral (ESPOL) bajo la dirección de James Zeidler y con fondos proporcionados por la OEA y la ESPOL. Dentro de los objetivos propuestos, comprendió la excava- ción arqueológica intensiva de áreas seleccionadas dentro del poblado de San Isidro, con el propósito de obtener una cronología precisa de las diferentes ocupaciones culturales que ocuparon la zona (Zeidler 1994).

Actualmente, una expedición de investigadores de la Universidad Federal del Extremo Oriente (Rusia) y la ESPOL, están llevando a cabo un análisis comparativo entre Valdivia y una cultura de la costa del Pacifico de Rusia para comparar la adaptación humana a los cambios ambientales en los lados opuestos del Pacífico (García 2015).

En este trabajo, se expone estadísticamente, la dependencia existente, entre la producción y el uso de la Cerámica Valdivia fase VIII1 o Complejo Piquigua. La forma de las vasijas será observada con relación a dos variables. Por una parte, el tamaño del desgrasante y por otra los acabados o tratamientos realizados en los recipientes.

\section{METODOLOGÍA}

La cerámica, como cualquier otro artefacto, representa la cultura material dejada por una sociedad en el pasado, pero además refleja los procesos Postdeposicionales, que actuaron una vez que el artefacto fue abandonado. Este artefacto tiene una forma, tamaño, textura, composición y localización en un espacio y tiempo (Barceló 2011:8) y fue producto de procesos de producción, distribución y uso (Ibíd.). En este caso, nos estamos refiriendo a la cerámica de la sociedad Valdivia Fase VIII.

El conjunto cerámico está formado por 368 fragmentos, entre bordes, bases y cuerpos de vasijas y provienen de tres tipos de contexto arqueológico: deposiciones ocupacionales, relleno de montículo y basural. De esta 
muestra se escogieron 104 fragmentos de bordes que representaron un diámetro mayor al $10 \%$ de la vasija.

Una «deposición ocupacional» es un contexto formado por el uso regular y constante de un espacio, por parte de un grupo de personas de una sociedad, en un tiempo dado (Jadán 1986). El contexto «relleno de montículo» se refiere a los depósitos encontrados en el montículo Cerro de la Cruz que fue rellenado con suelos arcillosos, mezclados con material cultural (ibíd.). El contexto «basural» se refiere a un área de actividad especializada donde son arrojados los objetos cuando se rompen o se desgastan y no son reprocesados y cuando se producen desechos inútiles (Shiffer 1972).

La cerámica fue caracterizada conforme a la metodología de «análisis modal», iniciada por Rouse (1940) y continuada por Lathrap (1962) entre otros investigadores. La metodología consistió en clasificar sucesivamente un complejo cerámico según diferentes dimensiones o magnitudes, dentro de los cuales se distinguió una serie de modos (Jadán 1986).

La magnitud se refiere a las propiedades observables reflejadas en la materialidad de los objetos resultado de la acción social (Barceló 2011). Los modos son definidos por referencia a las magnitudes y son unidades mínimas de variación socialmente significativas, es decir, representan la consecuencia material de una acción social. No obstante, no se estudia la causa de la acción individual, sino las causas de la acción colectiva, es decir, la repetitividad de las actividades de trabajo de los hombres y mujeres y el grado de regularidad que manifiestan las consecuencias materiales de dichas actividades y para estudiar esta variabilidad utilizaremos el análisis estadístico (ibíd.).
En este análisis, se consideran las siguientes dimensiones o magnitudes: Forma, textura (tamaño de desgrasante) y acabado de superficie.

\section{RESULTADOS Y DISCUSIÓN}

\section{La forma y el acabado de superficie de las vasijas Valdivia Fase VIII}

Hace referencia a los criterios morfológicos de las vasijas en relación con el acabado de sus superficies. Para definirla se ha utilizado los conceptos planteados por Shepard (1971:225-254), quien basó su clasificación en la definición de los puntos de contorno o puntos característicos definidos por Birkhoff (1933). La aplicación de estos conceptos permite reconstruir la morfología de las vasijas y definirlas conforme a la simetría, estructura, tipo de contorno, forma geométrica y proporción.

De acuerdo con estos criterios geométricos (Shepard 1971:225-254), se observaron tres clases estructurales de vasijas en el complejo Valdivia terminal: Vasijas No Restringidas (VnoR); Vasijas Restringidas Simples y Dependientes (VRSD) y Vasijas Restringidas Independientes (VRI). Estas clases estructurales, a su vez corresponden a dieciséis categorías funcionales, distribuidas de la siguiente manera: Siete formas funcionales dentro de las Vasijas No restringidas, cuatro formas para las Vasijas Restringidas Simples y Dependientes y finalmente cinco formas funcionales para las Vasijas Restringidas Independientes (Figuras 1-2).

Para definir las categorías funcionales de la vajilla, se reagruparon artefactos ligados entre sí por la producción, la función-forma, uso/consumo. Las categorías funcionales se definieron por la recurrencia y asociación de Modos dentro de las dieciséis formas ya mencionadas, considerando, que es el grado de aceptación social que tuvieron los modos, lo que determinó la recurrencia de los 
mismos en la vajilla Valdivia (Jadán 1986:401). En este sentido, todo objeto socialmente producido funciona como símbolo o indicador de una realidad social que está definida, precisamente por la acción colectiva, es decir, la capacidad del grupo social para producir y reproducirse (Barceló 2011).

Las Vasijas no restringidas (VNoR), se encuentran en los recipientes de contornos simples con puntos terminales, comprenden las siguientes formas: Plato elipsoide poco profundo de paredes abiertas, plato elipsoide de profundidad media de paredes abiertas y plato esferoidal profundo. Morfológicamente se caracterizan por tener bordes evertidos o evertidos engrosados al interior, labios redondeados o romos (esto últimos sirven como campo decorativo).

Las técnicas de acabado consisten en superficies pulidas y sin brillo, efecto producido cuando se pule superficies no suficientemente secas (Shepard 1971:123, 190). La decoración muestra patrones lineales simples o patrones de bandas triangulares. Todos estos diseños están efectuados con la técnica del grabado, con instrumentos de punta fina cuando la arcilla está seca o en diversos momentos del secado. El interior del borde o los labios sirven de campo de diseño.

Algunos fragmentos de bases de forma anular, encontrados en estos contextos, nos sugieren que fueron para servir alimentos o para su enfriamiento. La base anular otorga mayor estabilidad a los recipientes (Figura 1a, 1b, 1d). Algunas vasijas presentan una variante morfológica- funcional, que consiste en una protuberancia central en el interior, probablemente algún tipo de tapa para proteger los alimentos (figura 1f) (Jadán 1986:344, 367).
Dentro de este grupo, también se encuentran, un pequeño cuenco profundo de borde engrosado; un cuenco esferoidal y un cuenco carenado de profundidad media y finalmente un cuenco con borde de contorno inflexionado. Formas que tienen los contornos no restringidos y que probablemente también se usaban para el mantenimiento de alimentos cocinados.

Un segundo grupo son las Vasijas Restringidas Simples y dependientes (VRSD), vasijas tipo cazuela, utilizadas seguramente para la conservación de alimentos ya cocidos. Se definieron las siguientes formas: Cuenco esferoidal poco profundo, cuenco esferoidal de profundidad media, cuenco carenado de profundidad media y cuenco esferoidal profundo.

Estas formas se caracterizan por tener bordes invertidos y labios romos, redondeados o simétricamente delgados. Las formas decoradas exhiben patrones de bandas triangulares, formadas por líneas cuidadosamente grabadas y encerradas por líneas horizontales que sirven como elementos divisorios. Estos patrones se realizan sobre superficies previamente pulidas o ahumadas y cubren la superficie externa de la vasija (Figura 1c, 1e).

Un tercer grupo, son las Vasijas restringidas independientes (VRI), con contornos inflexionados y las Vasijas restringidas Independientes con contornos complejos. Dentro de las primeras, se distinguen las siguientes formas: Olla esferoidal con cuello corto, olla esferoidal con cuello campaniforme, olla esferoidal de borde evertido horizontal y olla elipsoide con cuello corto directo o recto (Figura 2g, 2k, 2h).

Los tres primeros tipos de vasijas se identifican por el borde evertido, labios de forma redondeada o romo. La bimodalidad en la 
forma del labio está relacionada con el aspecto funcional $\mathrm{y} / \mathrm{o}$ decorativo de la vasija; posiblemente evitan que el líquido se derrame fácilmente; en otros casos, sirven como campo decorativo.

Los contornos esferoidales y bases redondeadas son más funcionales, por cuanto son resistentes al choque térmico durante el proceso de cocción y enfriamiento (Rye 1981:27). Las superficies de estas vasijas presentan rugosidades o alisados en la parte externa y un revestimiento de engobe al interior del borde, técnica relacionada con la función.

Como estas vasijas generalmente son utilizadas en la cocción de alimentos, no se las sella en el exterior, porque el fuego destruye el revestimiento, en cambio, el interior del labio del borde es sellado, para evitar residuos de comida permanente (Ibíd.). Estas formas además, están decoradas con patrones lineales simples, como puntuaciones con caña en el labio, o pequeñas líneas inclinadas en el cuello.

Otro diseño son las impresiones, hechas con un instrumento de punta triangular de tama- ños diferentes, ubicadas de manera lineal y paralela en el cuerpo de la vasija. Frecuentemente este tipo de técnica combina la técnica de modelado que consiste en presionar la arcilla cuando es plástica con un instrumento suave, formando medallones o tiras largas a manera de relieve en la superficie externa (Rye 1981:92).

Un arreglo no muy frecuente es el de bandas de líneas inclinadas, que consisten en incisiones hechas cuando la arcilla está húmeda. Este tipo de diseño se encuentra en la olla elipsoide con cuello corto directo o recto. Contrario a las anteriores, esta si está finamente acabada, tiene superficies engobadas y decorada con patrones de bandas triangulares, o en arreglos de círculos o semicírculos, todos realizados con la técnica del grabado, es decir, cuando la pasta está seca (Figura 2h).

Además, dentro de las vasijas Restringidas Independientes, están las de contorno complejo, cuya variante es la Olla con borde carenado y cuello constreñido (2j). Morfológicamente se caracteriza por tener borde invertido, labios romos o de filos redondeados. Exhiben diseños de patrones triangula-

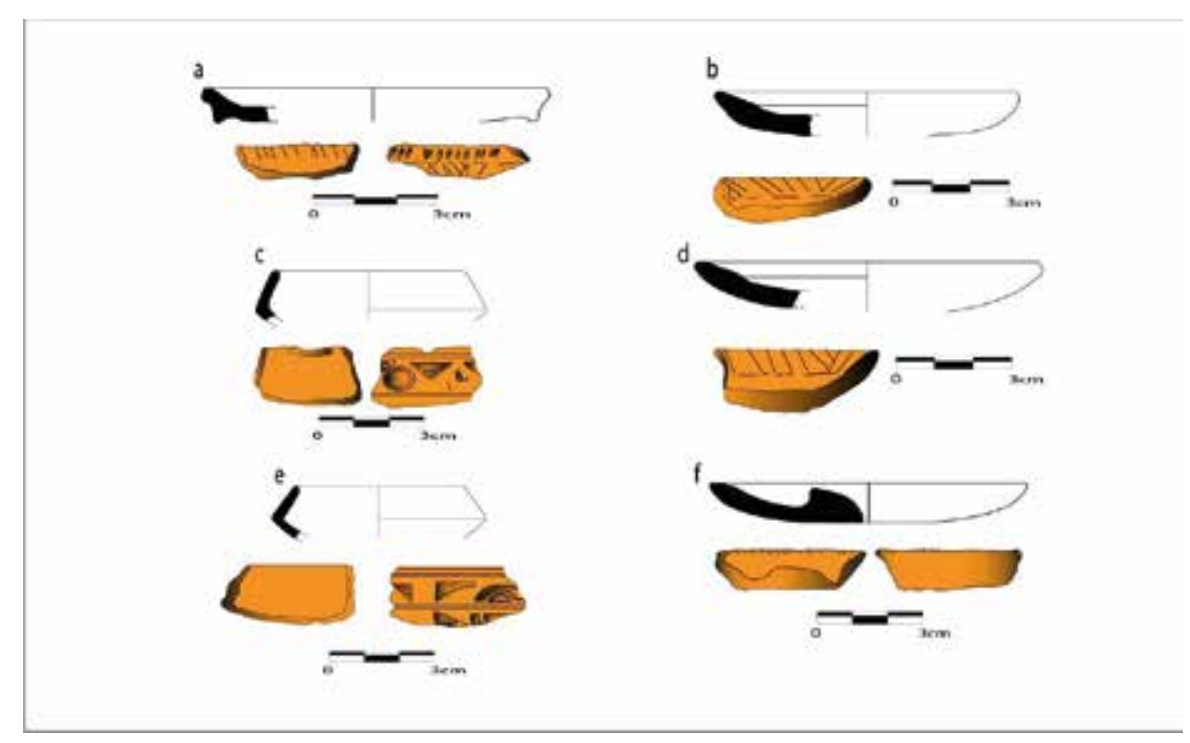

Figura 1: Vasijas No Restringidas (a, b, d, f) y Vasijas Restringidas Simples y Dependientes (c y e) Fuente: elaboración propia. 


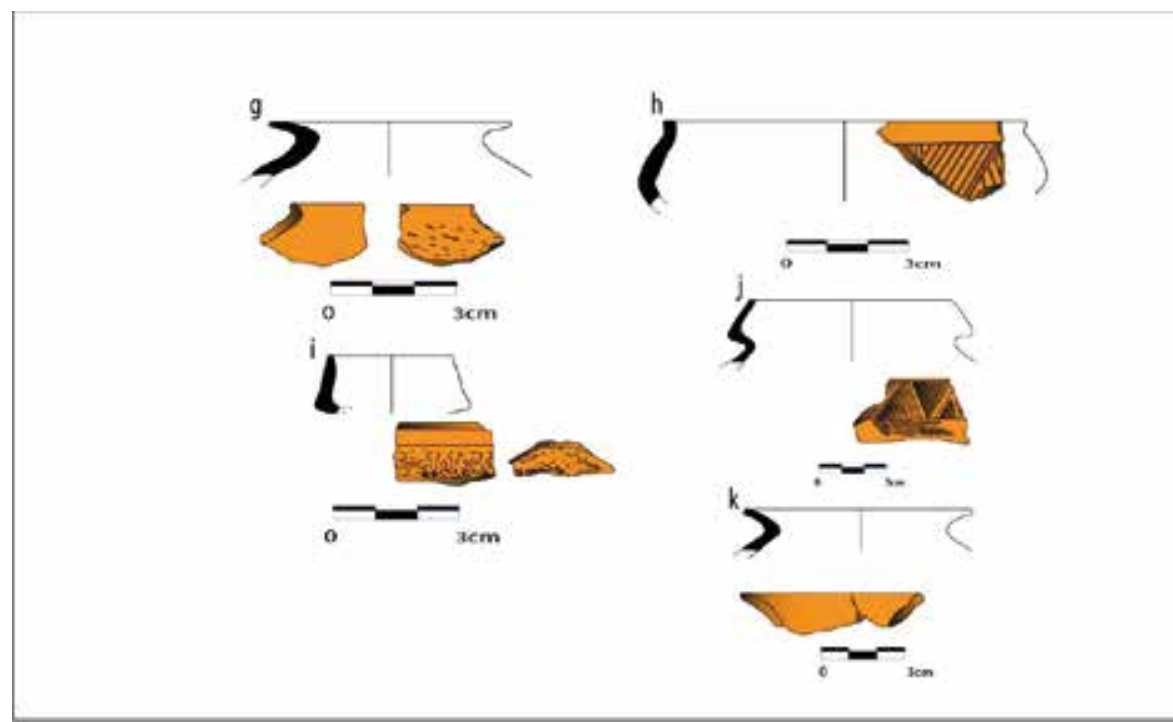

Figura 2: Vasijas Restringidas Independientes: contornos inflexionados ( $\mathrm{g}$, $\mathrm{k}$ y h) y contornos complejos (j, i)

Fuente: elaboración propia.

res, similares a las formas ya explicadas, realizados sobre superficies pulidas $\mathrm{o}$ ahumadas. Esta forma de acabado, evidentemente nos indica, que no fueron utilizadas para cocer alimentos, se presenta con cuello estrecho y son las botellas cerámicas, similares a las encontradas por Staller (1991) en el sitio la Emerenciana de la Provincia de El Oro (figura 2i).

\section{El tamaño de desgrasante en las vasijas Valdivia Fase VIII}

Las propiedades observables en la pasta proporcionan información sobre la materia prima que las o los ceramistas obtuvieron de la naturaleza, como arcilla, tipo y tamaño de desgrasante, combustible y otros componentes. Permite además inferir las técnicas de manufactura de la vasija y las variaciones tecnológicas.

Hay que tener presente, que los desgrasantes son sustancias no plásticas que se agregan intencionalmente a la pasta, o que están contenidas en forma natural en la arcilla. Las razones por las que los o las alfareras utilizan desgrasantes, son para controlar la plasticidad, servir como ligamento durante el proceso de secado o, reducir las tensiones internas que resultan del encogimiento de la arcilla durante el proceso de quemado (Shepard 1971; Rye 1981; Braun 1981).

En la cerámica del complejo Piquigua, se identificaron tres tamaños de desgrasantes o inclusiones. Las inclusiones de tamaño fino tienen entre $0.10 \mathrm{~mm}$ a $0.25 \mathrm{~mm}$, las de grano medio entre $0.25 \mathrm{~mm}$ a $0.50 \mathrm{~mm}$ y las de grano grueso, entre $0.85 \mathrm{~mm}$ a $2.0 \mathrm{~mm}$. Los tamaños fino y grueso comprometen, cada uno, a un $39 \%$ de las muestras, quedando un $22 \%$ de la muestra con una pasta con desgrasante de tamaño medio, que no es exclusividad para ninguna de las formas, a excepción de las ollas esferoidales (VRI), donde un $35 \%$ de las mismas presenta este tamaño de inclusiones.

La pasta con desgrasante de tamaño fino se encuentra en las vasijas comúnmente llamados platos (VnoR) cuencos, ollas elipsoides con cuello corto y ollas con borde carenado y cuello constreñido (VRSD), estos recipientes no son utilizados para cocer alimentos. La cerámica, con este tipo de pasta, tiene desgrasantes compuestos por 
feldespatos, cuarzo, anfíboles, opacos, óxidos de hierro y pirógenos; solamente dos muestras presentaron restos orgánicos como raíces.

Esta pasta tiene un tamaño muy uniforme, lo que plantea la posibilidad que el desgrasante, fue un componente de la arcilla (Véase Shepard 1971:161), es decir, la ceramista disponía de una fuente donde obtenía la arcilla; otra posibilidad, es que la refinaba mediante la utilización de tejidos (Marcos 1973).

La textura gruesa encontrada en las ollas de cocción (VRI), está compuesta por los mismos minerales de la pasta de textura fina, pero además tiene fragmentos de roca desgastados y erosionados, estos, difícilmente se mezclan con las arcillas por procesos naturales (Shepard 1971:25-29).

Lo que sugiere una acción intencional de los o las ceramistas Valdivia al añadir ciertos tipos de desgrasante a la pasta para la confección vasijas, como el caso de las vasijas de uso ordinario utilizadas en la cocción de alimentos. La cerámica con desgrasantes grueso tiene más resistencia a la propaga- ción de las fracturas que las pastas con desgrasante fino, en las cuales las fracturas se expanden más rápidamente (Braun 1982, Rye 1981).

Los detalles observados tanto en el estudio microscópico de la pasta, como en el tratamiento de superficie de las vasijas, explicados en este estudio, sugieren que hubo una asociación, entre el tamaño de desgrasante utilizado, acabado de superficie y cierto tipo de vasijas, que implicaría en última instancia, una relación con la función de cada artefacto. Relaciones de dependencia que ahora interesan analizar con los estadísticos presentes en el programa PAST.

\section{El uso de la estadística entre tamaño del desgrasante y forma de la vasijas}

Debido a que necesitamos conocer y comprobar la relación de dependencia o no entre una variable cualitativa y otra cuantitativa, vamos a aplicar el estadístico denominado Tablas de Contingencia:

Usaremos el estadístico Tabla de Contingencia para medir la relación entre el tamaño de desgrasante y la forma de vasija. «La frecuencia de aparición de estas variables

Tabla 1: Tabla de Contingencia entre formas de vasijas y tamaño de desgrasantes utilizado

\begin{tabular}{|c|c|c|c|c|}
\hline Tipos & Fino & Medio & Grueso & Total \\
\hline VnoR & 21 & 5 & 2 & 28 \\
\hline VRSD & 13 & 1 & 0 & 14 \\
\hline VRI & 7 & 16 & 39 & 62 \\
\hline TOTAL & 41 & 22 & 41 & 104 \\
\hline
\end{tabular}

Fuente: elaboración propia. 
más que resúmenes de una variable cualitativa, es una variable cuantitativa que puede usarse para afirmar la intensidad de la propiedad» (Barceló 2011:328). Los desgrasantes en la pasta de los recipientes Valdivia, se ha caracterizado como: fino, mediano y grueso. Se plantea la hipótesis nula y alterna:

$\mathrm{H}_{0}$ : La forma de las vasijas y el tamaño de los desgrasantes son independientes.

$\mathrm{H}_{1}$ : Los dos criterios no son independientes.

Se compara lo observado representado en la Tabla de Contingencia con un «Modelo Esperado». Las variaciones del tamaño de desgrasante encontrado en la pasta, constituyen la expresión visible de las consecuencias materiales de una acción social (variable dependiente o efecto observado) que no tiene por qué ser la misma en las diferentes formas de vasijas (factor o causa probable).

El problema arqueológico a responder sería: «las necesidades por tener diferentes tipos de vasijas para diferentes funciones de la cerámica Valdivia terminal, afectan la variabilidad de los artefactos, como consecuencia de las decisiones del artesano o artesana para añadir tal o cual tamaño de desgrasantes a la pasta con la que confecciona las vasijas»» El resultado obtenido es:

$\mathrm{M} ; \mathrm{N}: 3 ; 3$

Degrees freedom: 4

$\mathrm{Chi}^{\wedge} 2: 55.244$

p (no assoc): $2.8879 \mathrm{E}-11$

Monte Carlo p: 0.0001

Crammer's V: 0.51536

Contingency C: 0.58899

La probabilidad de no asociación ( $\mathrm{p}$ no assoc) obtenida es igual a 2.8879 E-11, es decir, menor a p005. Por lo tanto, las dos variables observadas no son independientes. Es decir, hubo una selección del o la cera- mista al elegir el tamaño de desgrasantes para cada forma o tipo de vasija, lo que estaría relacionado con la producción y la intención de funcionalidad de las vasijas.

Aplicando la prueba Monte Carlo observamos que solamente hay una diferencia. Por otra parte, la prueba de Cramer's V, es una medida de la intensidad de la relación que se basa en $\chi 2$ cuadrado, tiene un valor entre $0 \mathrm{y}$ 1 y puede alcanzar el 1 para tablas de cualquier dimensión (id.). En este caso alcanza un valor de 0.51536 , lo que demuestra una correlación relativamente intensa entre las dos variables, es decir las dos variables están asociadas y hay una dependencia directa superior a la mitad del valor definido para este estadístico (entre 0 y 1 ).

El coeficiente de contingencia (Contingency C) es otra medida de asociación basada en $\chi$ 2. (ob. cit.). En este caso tiene un valor de 0.58899 , es decir, la dependencia existente entre el tamaño del desgrasante y la forma de la vasija es verdadera, en la cual, conforme sea la forma-función de la vasija, cambia el tamaño del desgrasante en forma significativa, con un valor de coeficiente de contingencia de C: 0.58899 .

Barceló (ibíd.), sugiere indagar todas las posibilidades para caracterizar la relación de dependencia, aunque esta relación sea baja. Así mismo, propone utilizar análisis multivariante como una técnica de interpretación de tablas de contingencia que, por definición, son bivariantes y no multivariantes (Ibíd.). Este método se llama análisis de correspondencia: 
Análisis de correspondencia entre Tamaño de desgrasante y Forma de Vasija

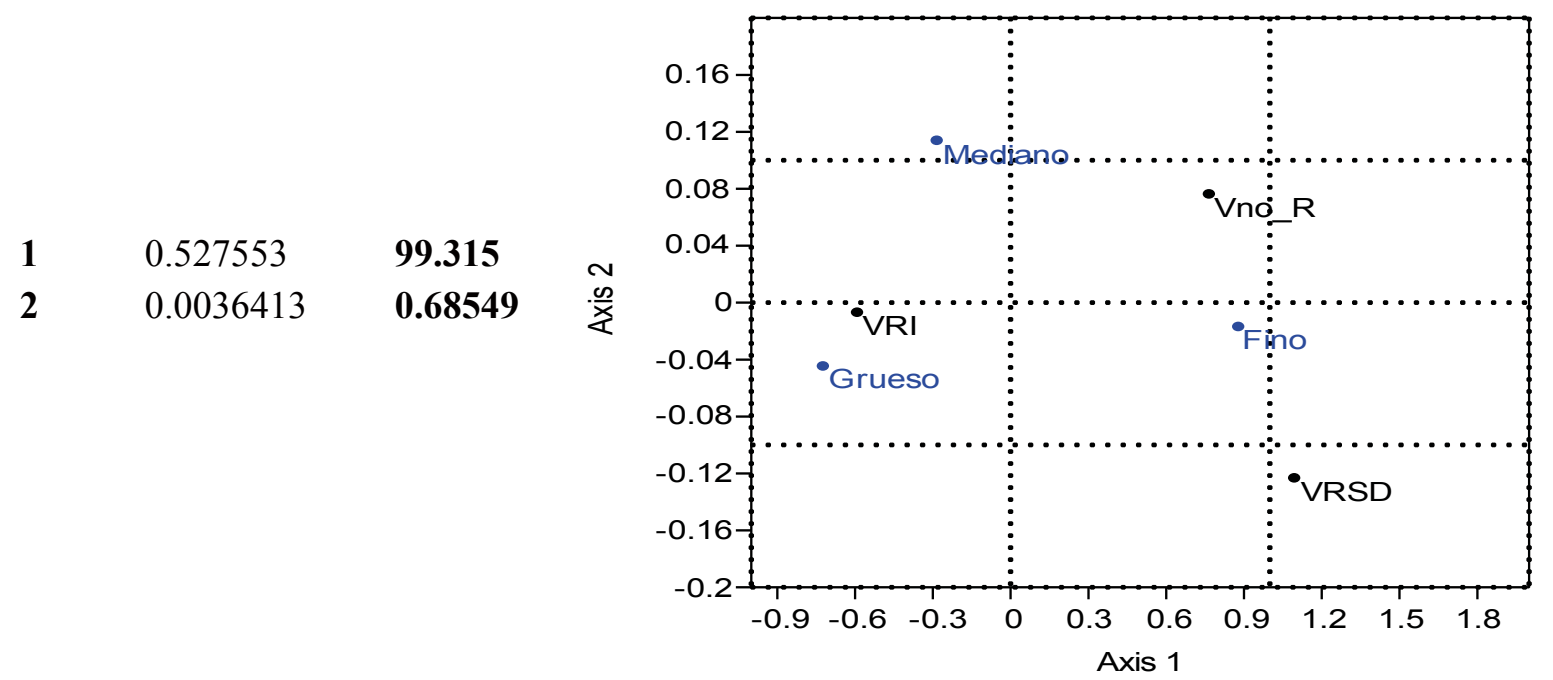

Con el análisis de correspondencia, se intenta relacionar el tamaño de desgrasante, con la forma de vasija. El grafico muestra tres tamaños diferenciados de desgrasantes. En el primer eje se ve claramente la variación. El estadístico separa hacia la izquierda del Eje Y, a las Vasijas Restringidas Independientes (VRI) y las asocia con desgrasante grueso, indicando que en ese caso, la relación es contundente. Mientras que el desgrasante fino es compartido por dos tipos de vasijas, las Vasijas No restringidas (VnoR) y la Vasijas Restringidas Simples y Dependientes (VRSD), que las sitúa a la derecha del eje Y. Observamos además que el desgrasante mediano se aleja discretamente de las tres formas de vasijas, no obstante, lo ubica a la izquierda del eje, interpretando como una no preferencia para ninguna de estas formas, aunque ligeramente más cercano a la Vasija Restringida Independiente.

\section{Aplicando los estadísticos en la relación al acabado de superficie y a la forma de vasija}

Se plantea la hipótesis nula y alterna:

Tabla 2: Tabla de Contingencia entre formas de vasijas y acabado de superficie

\begin{tabular}{|c|c|c|c|c|c|c|}
\hline Tipos & Rugoso & Alisado & Pulido & Ahumado & Engobe & Total \\
\hline VnoR & 0 & 9 & 45 & 10 & 2 & 66 \\
\hline VRSD & 0 & 5 & 38 & 25 & 2 & 70 \\
\hline VRI & 26 & 31 & 21 & 25 & 53 & 156 \\
\hline Total & 26 & 45 & 104 & 60 & 57 & 292 \\
\hline
\end{tabular}

Fuente: elaboración propia. 
H0: La forma de la vasija y el acabado de superficie de las vasijas son independientes H1: Los dos criterios no son independientes.

Se compara lo observado representado en la Tabla de Contingencia 2, con un «Modelo Esperado». El problema arqueológico a responder sería: «si las necesidades por tener diferentes tipos de vasijas para diferentes funciones en la sociedad Valdivia, afectan la variabilidad de los artefactos, como consecuencia de las decisiones del artesano o artesana, para realizar diferentes tipos de acabado de las superficies de las vasijas ».

El resultado obtenido es:

M; N: 3; $5 \quad$ Degrees freedom: 8

Chi ^2: 122.09

p (no assoc): $1.2255 \mathrm{E}-22$

Monte Carlo p: 0.0001

Crammer's V: 0.45723

Contingency C: 0.542999
Observamos que la probabilidad de que $\mathrm{H} 0$ sea verdadera con 8 grados de libertad es menor a 0.005 , es decir, es $1.2255 \mathrm{E}-22$. Por lo tanto, rechazamos $\mathrm{H}_{0}$ y concluimos que la forma de la vasija y el acabado de su superficie son dependientes en la cerámica Valdivia fase VIII. El test de Monte Carlo encuentra una diferencia. El análisis de la Crammer' V alcanza un valor cercano a 0.50 , demuestra que hay diferencias significativas entre las dos variables y que por tanto están asociadas.

Con el análisis de correspondencia intentamos relacionar el Acabado de Superficie de las vasijas con la Forma de las mismas. Existe cinco tipos de acabados utilizados representados en el gráfico: Rugoso, Alisado, Engobe, Pulido y Ahumado. El gráfico presenta claramente que la relación entre forma de vasija y acabado es contundente. En el primer eje se agrupa a las vasijas Restringidas Independientes (VRI) asociadas a tres modos Rugoso, Engobe y Alisado.

En lo que respecta al Análisis de correspondencia para Acabado de superficie y Forma de Vasija, tenemos:

$\begin{array}{lll}\mathbf{1} & 0.384862 & \mathbf{9 2 . 0 4 5} \\ \mathbf{2} & 0.0332627 & \mathbf{7 . 9 5 5 2}\end{array}$

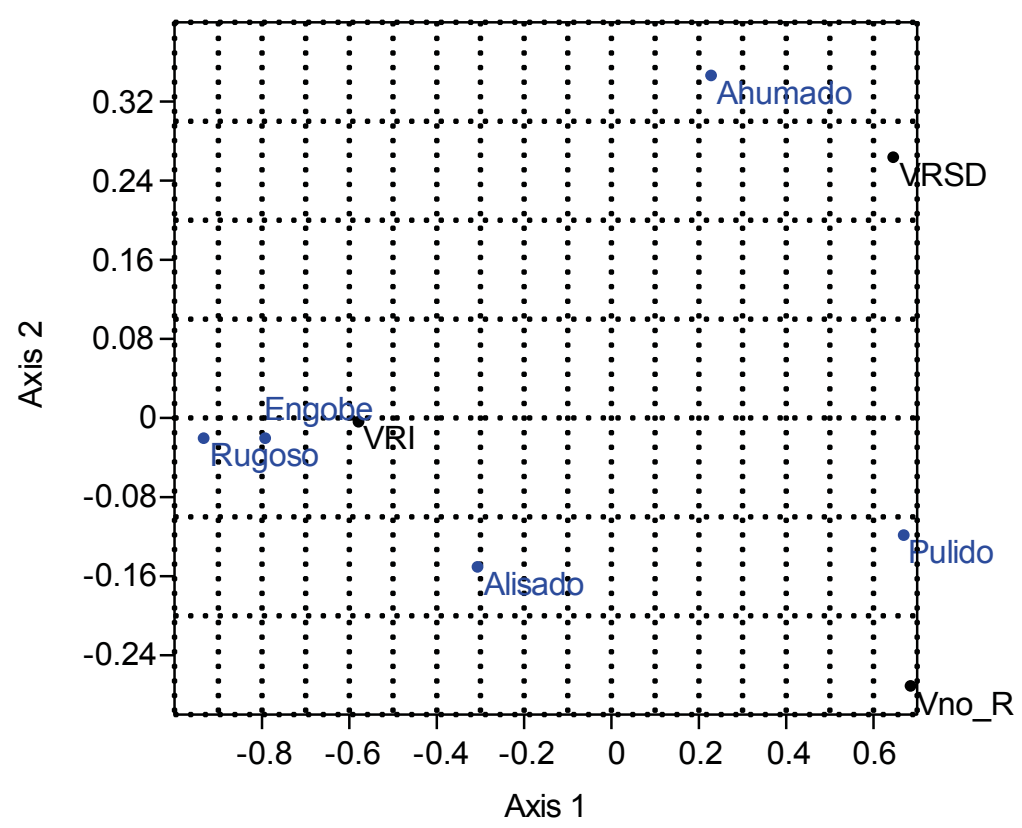


Mientras tanto, el modo pulido, a la derecha del eje $\mathrm{Y}$, está discretamente cercano a las Vasijas No Restringidas (VnoR) y el modo ahumado en las Vasijas Restringidas Simples y Dependientes (VRSD).

\section{CONCLUSIONES}

La utilización de estadísticos, en este caso, nos ayuda a mirar la relación existente entre morfología y tamaño de desgrasante; entre morfología y acabado de superficie. En el primer caso, hay una relación de dependencia entre las dos variables. En el segundo, la relación es contundente. Para los datos obtenidos hasta la actualidad, los resultados demostraron que hay una intención en los artesanos o artesanas Valdivia Fase VIII para realizar cierto tipo de vasijas con un tipo específico de tamaño de desgrasante y que cierto acabado corresponde asimismo, a cierta forma y función del recipiente. De tal manera que existe una propósito entre la producción de la cerámica y el uso de la misma.

\section{REFERENCIAS \\ BIBLIOGRÁFICAS}

Barceló, J. (2011). Análisis de Datos Arqueológicos Teoría y Métodos. Barcelona, España: Universidad Autónoma de Barcelona (Documento inédito).

Birkhoff, G. (1933). Aesthetic Measure. Massachusetts, Estados Unidos: Harvard University Press.

Estrada, E. (1956). Valdivia, un Sitio Arqueológico Formativo en la Costa de la Provincia del Guayas. Guayaquil, Ecuador: Museo Víctor Emilio Estrada.

Jadán, M. (1986). La Cerámica del Complejo Piquigua (Fase VIII) de la cultura Valdivia en San Isidro Norte de Manabi: Un análisis modal (tesis de pregrado inédita). Escuela Superior Politécnica del Litoral ESPOL, Guayaquil, Ecuador.

Lathrap, D. (1962). Yarinacocha: Stratigraphic Excavation in the Peruvian Monta$n a$ (tesis Doctoral inédita) Departamento de Antropología, Universidad de Harvard, Cambridge, Massachusetts, Estados Unidos.

Lathrap, D., Marcos J. \& Zeidler J. (Mayo de 1977). Real Alto: an ancient ceremonial center. Archaeology 30 (1), pp. 2-13.

Marcos, J. (1973). Tejidos hechos en telar en un contexto Valdivia Tardío. Separata de Cuadernos de Historia y Arqueología, Casa de la Cultura Ecuatoriana, Año XXIII, (40).

Meggers, B., Evans, C., \& Estrada, E. (1965). The Early Formative Period of Coastal Ecuador: The Valdivia and Machalilla Phases. Washington DC, Estados Unidos: Smithsonian Contributions to Anthropology 1.

Norton, P. (1982). Preliminary observations on Loma Alta, an early Valdivia midden in Guayas Province, Ecuador. En J. Marcos \& P. Norton. Primer Simposio de Correlaciones Antropológicas Andino-Mesoamericano (pp. 101 - 109). Escuela Superior Politécnica del Litoral, Guayaquil, Ecuador. 


\section{Unach}

Rye, O. (1981). Pottery Technology. Washington DC, Estados Unidos: Taracum Inc.

Rouse, I. (Octubre-Diciembre-1940). Prehistory in Haití: a STudy in Method. Anthropology (21), 673-675.

Shiffer, M. (Abril de 1972). Archaeological context and systemic context. American Antiquity, 37 (2), 156-165.

Staller, J. (1996). El sitio Valdivia Tardio de la Emerenciana en la Costa sur del Ecuador y su significación del desarrollo de Complejidad en la Costa oeste de Sudamérica. Cuadernos de Historia y Arqueología, (46-47), 14-37.

Zevallos, C. \& Holm O. (1960). Excavaciones arqueológicas en San Pablo: informe preliminar. Guayaquil, Ecuador: Casa de la Cultura Ecuatoriana. Núcleo del Guayas.

Zevallos, C. (1971). La Agricultura en el Formativo Temprano del Ecuador (Cultura Valdivia). Guayaquil, Ecuador: Casa de la Cultura Ecuatoriana. Núcleo del Guayas.

Zeidler, J. (1994). Investigaciones Arqueológicas en el Valle Medio del Rio Jama. En J. Zeidler \& D. Pearsall (eds.). Arqueología Regional del Norte de Manabi, Ecuador Volumen I (pp. 72-98). Pittsburgh, Estados Unidos y Quito, Ecuador: Universidad de Pittsburgh y Ediciones Libri Mundi.
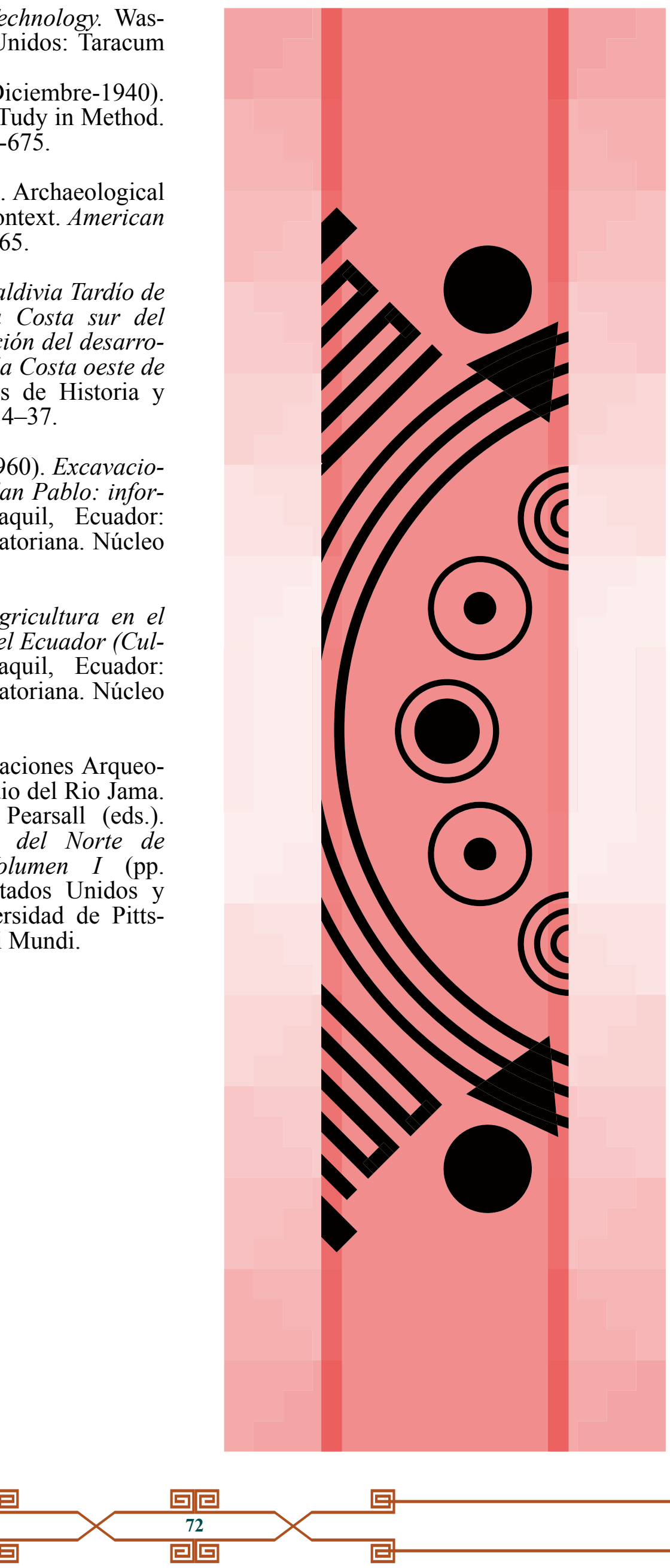